\title{
Sesamin and Hepatic Metabolites Derived from Sesamin and Episesamin Antagonize Farnesoid X Receptor and Reduce the Expression of Gluconeogenesis-Related Genes
}

\author{
Takashi SASAKI ${ }^{1, *}$, Mako OKUDA ${ }^{1}$, Tzu-Wen Hong $^{1}$, Yuichi WATANABE $^{1}$, Yu TAKAHASHI ${ }^{1}$, \\ Makoto SHIMIZU ${ }^{2}$, Yoshio YAMAUCHI ${ }^{1,3}$ and Ryuichiro SATO ${ }^{1,2,3, * *}$ \\ ${ }^{1}$ Food Biochemistry Laboratory, and ${ }^{2}$ Nutri-Life Science Laboratory, Department of Applied Biological \\ Chemistry, Graduate School of Agricultural and Life Sciences, The University of Tokyo, \\ Tokyo 113-8657, Japan \\ ${ }^{3}$ AMED-CREST, Japan Agency for Medical Research and Development, Tokyo 100-0004, Japan
}

(Received September 25, 2021)

\begin{abstract}
Summary Sesamin and episesamin are the main lignans found in refined sesame oil and have been reported to exert various health benefits. However, the health benefits of these lignans and their molecular mechanisms have not been fully understood. This study evaluated the effects of sesamin, episesamin, and their metabolites on the nuclear bile acid receptor, farnesoid X receptor (FXR, NR1H4), which regulate gene expression involved in bile acid metabolism and gluconeogenesis. By using two different cell-based luciferase reporter assay systems, we found that sesamin, sesamin metabolites, and some episesamin metabolites inhibited FXR activation driven by a bile acid and a synthesized agonist, and it is suggested that these compounds exert their antagonist activity by competing with the FXR agonists on the ligand-binding domain. Sesamin and its major metabolite SC-1 suppressed the expression of several gluconeogenesis-related genes governed by FXR in HepG2 cells but did not affect the expression level of CYP7A1, the rate-limiting enzyme for bile acid synthesis. Dietary sesamin supplementation (AIN-93G supplemented with $0.5 \%$ sesamin) led to the decreased hepatic expression of several gluconeogenesis-related genes and reduced blood glucose levels in mice, without adverse effects on bile acid metabolism. These results shed light on the health benefits of taking sesamin and episesamin.
\end{abstract}

Key Words sesamin, episesamin, farnesoid X receptor, gluconeogenesis, bile acid

The farnesoid X receptor (FXR, NR1H4) is a nuclear receptor activated by bile acids and highly expressed in the liver, ileum, kidney, and adrenal glands (1). FXR regulates the expression of multiple genes involved in bile acid homeostasis in the liver and small intestine. The small heterodimer partner (SHP), an FXR target, inhibits hepatocyte nuclear factor 4 (HNF4) alpha- and liver receptor homolog 1-mediated transcription of cholesterol $7 \alpha$-hydroxylase (CYP7A1), a rate-limiting enzyme in the classic bile acid synthesis pathway (2-5). It suppresses bile acid synthesis and protects the liver from bile acid-induced toxicity. Furthermore, FXRinduced SHP suppresses the expression of both ratelimiting enzymes in gluconeogenesis, phosphoenolpyruvate carboxykinase (PEPCK) and glucose 6-phosphatase (G6Pase) (6). Since elevated blood glucose levels in type 2 diabetes are partially due to increased gluconeogenesis in the liver $(7,8)$, administering FXR agonist GW4064 to $\mathrm{db} / \mathrm{db}$ mice alleviates gluconeogenesis and improves hyperglycemia (9). In contrast, it was also reported that treatment of cultured hepatoma cells or rat and mouse primary hepatocytes with FXR agonists

\footnotetext{
*,**To whom correspondence should be addressed:

*E-mail: atsasaki@g.ecc.u-tokyo.ac.jp

**E-mail: roysato@g.ecc.u-tokyo.ac.jp
}

increases the mRNA levels of G6Pase and PEPCK. This response is inhibited by FXR antagonists such as guggulsterone (GGS) and HS218 $(10,11)$. The repression of these gluconeogenesis-related genes by FXR antagonists is achieved by inhibiting the recruitment of FXR to the peroxisome proliferator-activated receptor gamma coactivator-1 alpha (PGC- $1 \alpha$ ) promoter and reducing its expression (11). As expected from these reports, treatment of FXR antagonists to $\mathrm{db} / \mathrm{db}$ mice and highfat diet (HFD)/streptozotocin-induced diabetic mouse models decreases the expression of gluconeogenesis-related genes and improves glucose homeostasis $(11,12)$. Therefore, FXR antagonists as well as FXR agonists are a promising target for improving glucose metabolism.

Sesamum indicum (sesame) seeds have many health benefits and are nutritionally, pharmacologically, and industrially important food (13). Sesamin, a lignan found abundantly in sesame seeds and oil, is a major bioactive component that ensures sesame's superior health benefits. For example, sesamin exerts anti-cancer (14) and hypotensive (13) activity, and improves lipid metabolism by reducing fatty acid synthesis, increasing fatty acid oxidation, and attenuating cholesterol synthesis (15). Episesamin, a stereoisomer of sesamin, is rarely found in unrefined sesame oil but is obtained from sesamin during the acid-clay bleaching in the oil 
refining process (16). There are fewer reports on the bioactivity of episesamin than those of sesamin. Still, it is known to have partially similar functions to sesamin in that episesamin activates fatty acid oxidase and inhibits lipogenic enzymes in the liver (17). Both sesamin and episesamin are converted into various metabolites by CYP1A2, CYP2C9, and catechol-O-methyltransferase (COMT) in the liver (18-21). Interestingly, the antioxidant effect of sesamin intake depends not on sesamin but on its metabolites, indicating that some of the health benefits of sesamin intake are exerted by its metabolites (18). Therefore, to clarify the health effects of sesamin and episesamin, it is important to study their metabolites.

We have previously constructed an assay system for measuring intracellular bile acid levels using a luciferase reporter gene driven by FXR activation. In the process of assessing the function of several food factors using this assay, it is found that sesamin reduces FXR activity without affecting intracellular bile acid levels. In line with this finding, we focused on the FXR antagonistic activity of sesamin and performed some experiments to clarify its novel physiological role. Here we find, for the first time, that sesamin and its metabolites and some episesamin metabolites antagonize the FXR. Sesamin reduced the expression level of gluconeogenesis-related genes by suppressing the FXR activity and lowers blood glucose levels in mice. These results indicate the novel health benefits of taking sesamin and episesamin.

\section{MATERIALS AND METHODS}

Chemicals. Sesamin, SC-1, SC-2, SC-1m, SC-2m, episesamin, EC-1-1, EC-1-2, and EC-2 came from Bioorganic Research Institute, Suntory Foundation for Life Sciences (Kyoto, Japan). GW4064 and taurolithocholic acid (TLCA) were purchased from Sigma-Aldrich (St. Louis, MO, USA). Chenodeoxycholic acid (CDCA) was purchased from FUJIFILM Wako Pure Chemical Corporation (Osaka, Japan).

Animal experiments. Animal experiments were performed with male C57BL/6J mice obtained from CLEA Japan, Inc. (Tokyo, Japan), following the Guidelines for the Care and Use of Laboratory Animals of the University of Tokyo, under the approval of the Animal Usage Committee of the University of Tokyo (Approval number P17-141).

Mice were housed with a 12:12-h light-dark cycle and provided water ad libitum and a standard diet (Labo MR Stock; Nosan Corp., Kanagawa, Japan) for the first week following their arrival. After acclimatization, the mice that had reached $11 \mathrm{wk}$ old were randomly assigned to one of the two experimental groups $(n=5$ each group), and the mice were fed a control diet (AIN93G) or a sesamin-supplemented diet (AIN-93G supplemented with $0.5 \%$ sesamin) for $1 \mathrm{wk}$. These mice were weighed on day 0 and day 7 . At the end of the experiment, the mice were euthanized under isoflurane anesthesia. The liver and serum samples collected from the facial vein using an animal lancet were stored at $-80^{\circ} \mathrm{C}$
A
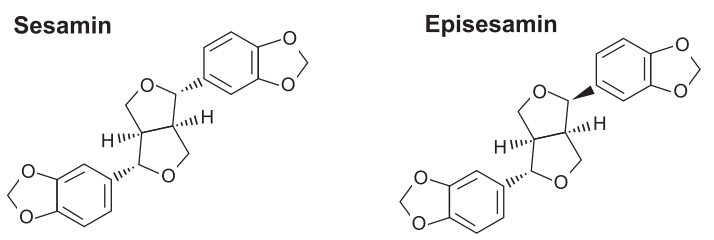

B

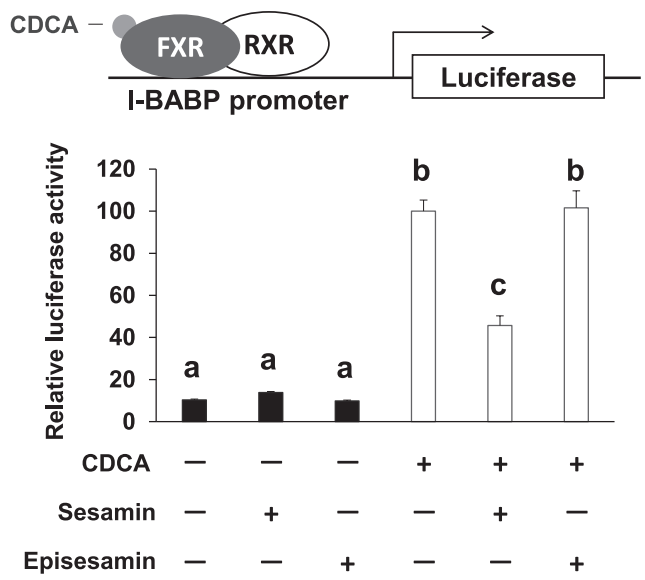

Fig. 1. Sesamin attenuates the CDCA-induced I-BABP promoter activation. (A) Structural representation of sesamin and episesamin. (B) HEK293T cells were transfected with FXR and RXR expression plasmids together with a human I-BABP promoter-luciferase plasmid. The cells were cultured with the CDCA $(50 \mu \mathrm{M})$, sesa$\min (50 \mu \mathrm{M})$, and episesamin $(50 \mu \mathrm{M})$ as indicated combinations for $24 \mathrm{~h}$. Then, a luciferase reporter assay was performed, normalizing against $\beta$-galactosidase activity $(n=3)$. The luciferase activity of the CDCA treated group was set at 100 . Data are mean \pm SD. Different letters above the bars indicate a significant difference $(p<0.05)$ according to one-way ANOVA (TukeyKramer post hoc test).

until further processing. Blood glucose and bile acid concentrations were determined using kits purchased from FUJIFILM Wako Pure Chemical Corporation.

Cell culture. HEK293T and HepG2 cells obtained from American Type Culture Collection (Manassas, VA, USA) were maintained in DMEM supplemented with 100 units/mL penicillin, $100 \mu \mathrm{g} / \mathrm{mL}$ streptomycin, and $10 \%$ FBS. The cells were incubated at $37^{\circ} \mathrm{C}$ under a $5 \%$ $\mathrm{CO}_{2}$ atmosphere.

Luciferase assay. HEK293T cells were plated in 24well plates at a density of $5.0 \times 10^{4}$ cells/well. After $24 \mathrm{~h}$, cells were transfected with $100 \mathrm{ng}$ of pCMV- $\beta$ Gal, an expression plasmid for $\beta$-galactosidase, $100 \mathrm{ng}$ of pME-FXR, an expression plasmid for human FXR, $100 \mathrm{ng}$ of pCMX-retinoid X receptor alpha $(\mathrm{RXR} \alpha)$, an expression plasmid for mouse $\operatorname{RXR} \alpha$, and $100 \mathrm{ng}$ of pGV-B2-intestinal bile acid-binding protein (I-BABP)-luciferase, a reporter plasmid with human I-BABP promoter region $(-862 /+30)$, for the determination of the ligand activity to full-length FXR. Alternatively, cells were transfected with $100 \mathrm{ng}$ of $\mathrm{pCMV}-\beta$-Gal, $100 \mathrm{ng}$ of pGAL4-FXR $\mathrm{LBD}_{\text {, an }}$ expression plasmid for GAL4 
A

Sesamin

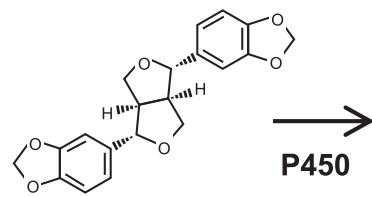

SC-1

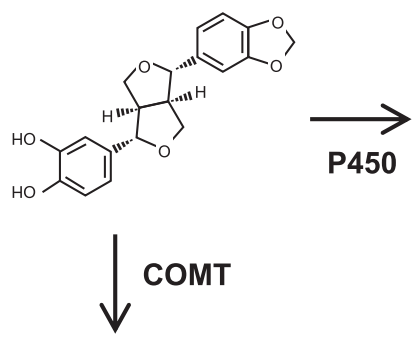

SC-2<smiles>Oc1ccc([C@H]2OC[C@]3(COC3c3ccc(O)c(O)c3)CO2)cc1</smiles>

SC-1m

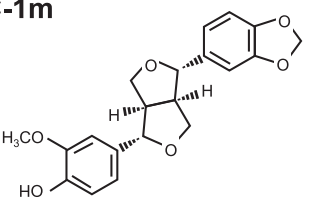<smiles>COc1ccc([C@@H]2OC[C@H]3C2OC[C@H]3c2ccc(O)c(O)c2)cc1O</smiles>

\section{B}
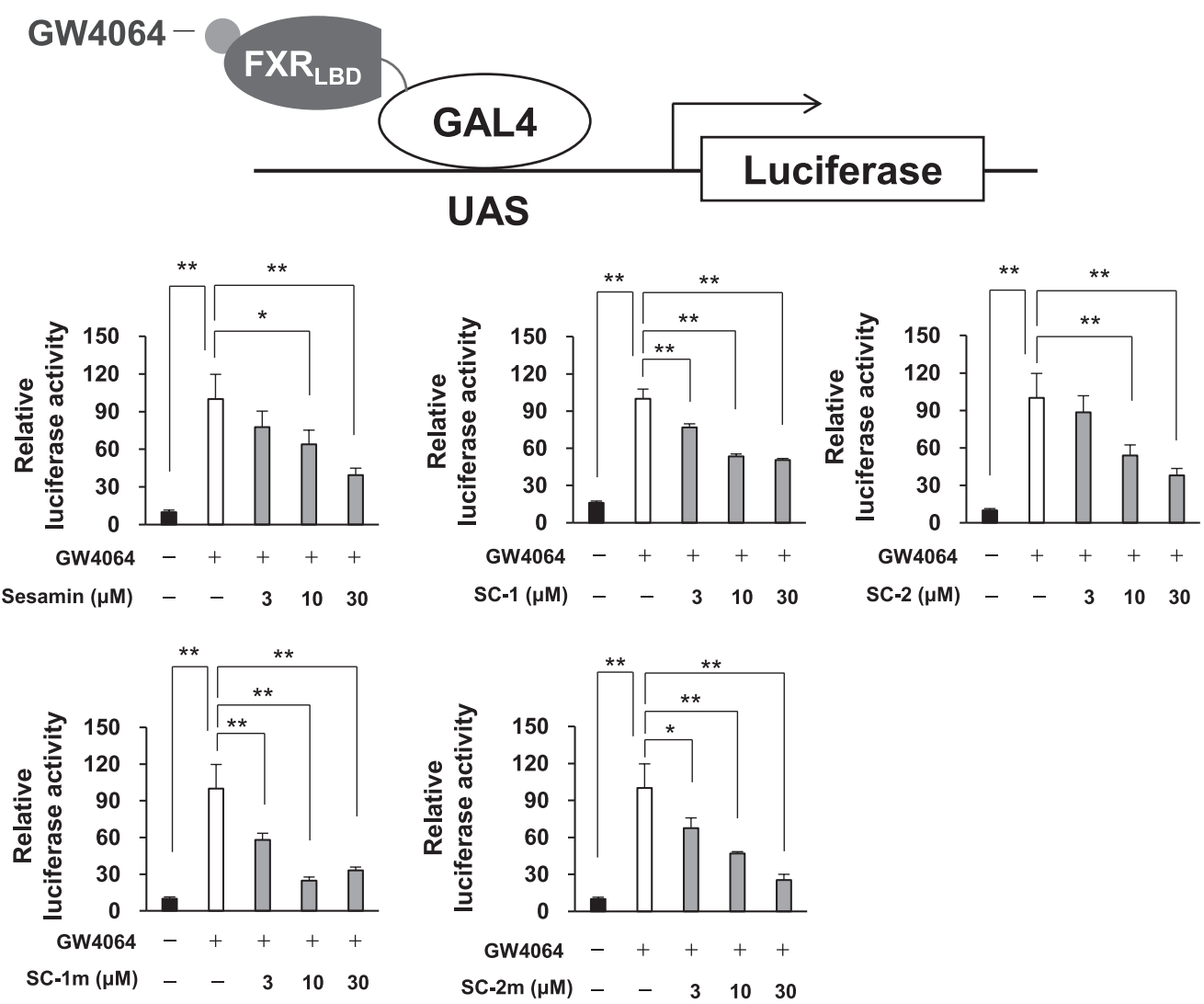

Fig. 2. Effects of sesamin and its metabolites on FXR activation by GW4064. (A) Chemical structures of sesamin and its

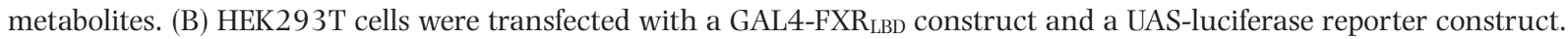
The cells were cultured with the GW4064 $(1 \mu \mathrm{M})$ and sesamin or its metabolites as the indicated concentration for $24 \mathrm{~h}$. Then, a luciferase reporter assay was performed, normalizing against $\beta$-galactosidase activity $(n=3)$. The luciferase activity of the GW4064 treated group was set at 100. Data are mean \pm SD. Statistical analyses were conducted using one-way ANOVA (Tukey-Kramer post hoc test). ${ }^{*} p<0.05 ;{ }^{* *} p<0.01$.

DNA-binding domain-FXR ligand-binding domain (193-472 aa) fusion protein, and $100 \mathrm{ng}$ of pUAS-luciferase, a reporter plasmid containing five copies of GAL4 binding sites, for the determination of the ligand activity to FXR ligand-binding domain. The medium was replaced $24 \mathrm{~h}$ later with DMEM containing penicillin/streptomycin, 10\% charcoal-stripped FBS, and indicated compounds or control vehicles. After $24 \mathrm{~h}$ of incubation, the luciferase and $\beta$-galactosidase activities were measured. For the determination of the ligand activity to TGR 5, HEK293T cells were transfected with $100 \mathrm{ng}$ of pCMV- $\beta$-Gal, $100 \mathrm{ng}$ of pCRE-luciferase, a reporter plasmid containing four copies of consensus sites for the cAMP response element (CRE), and 100 ng of pcDNA-TGR5, an expression plasmid for human TGR 5. The medium was replaced after $24 \mathrm{~h}$ with DMEM containing penicillin/streptomycin, 10\% charcoalstripped FBS, and indicated compounds or control vehi- 
A
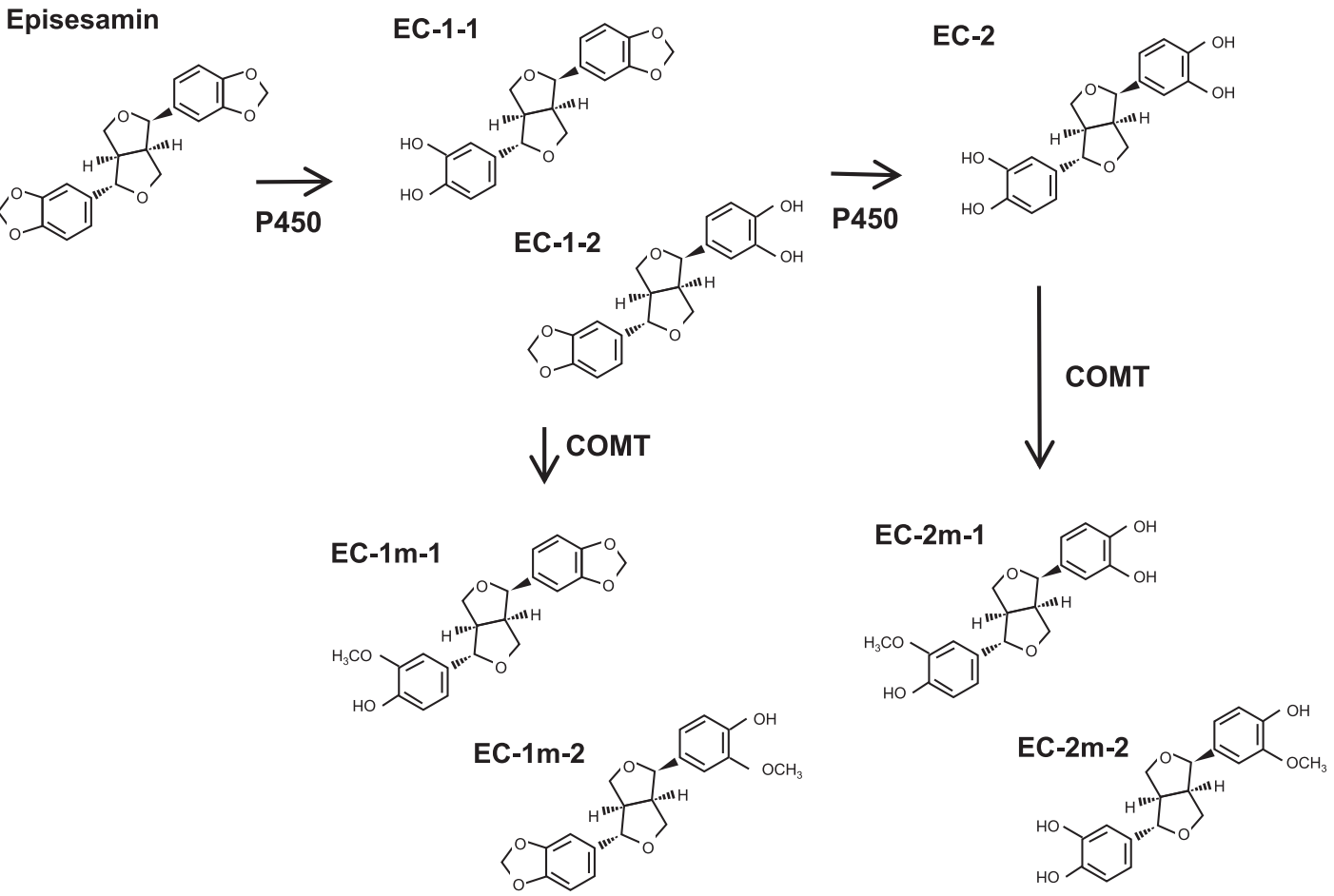

B
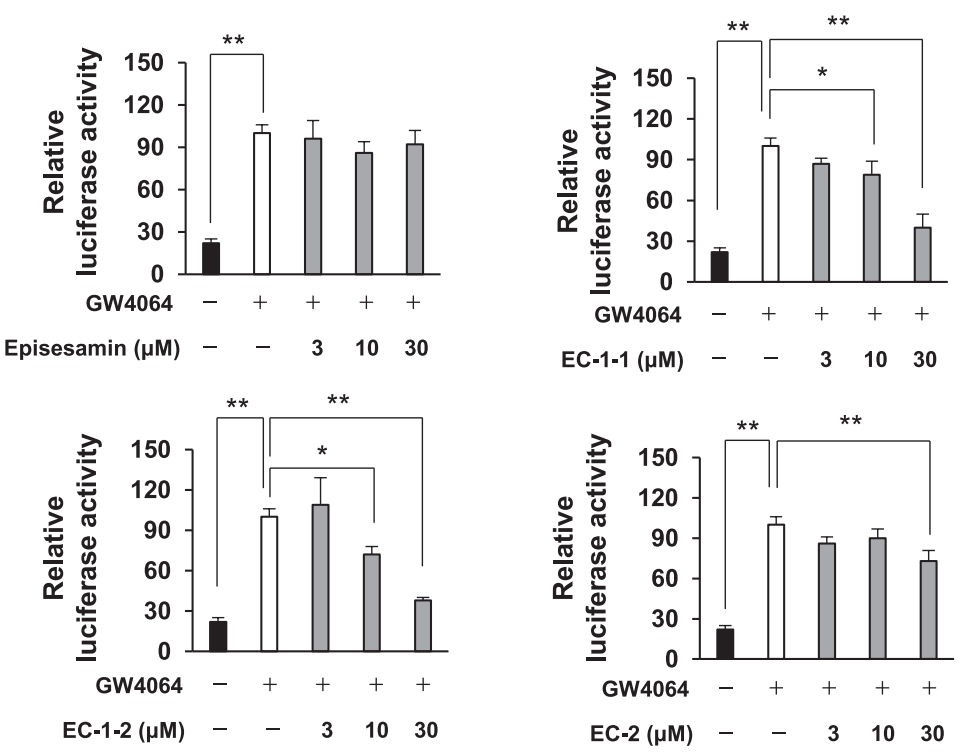

Fig. 3. Effects of episesamin and its metabolites on FXR activation by GW4064. (A) Chemical structures of episesamin and its metabolites. (B) HEK293T cells were transfected with a GAL4-FXR $\mathrm{LBD}_{\mathrm{L}}$ construct together with a UAS-luciferase reporter construct. The cells were cultured with the GW4064 $(1 \mu \mathrm{M})$ and either episesamin or its metabolites as the indicated concentration for $24 \mathrm{~h}$. Then, a luciferase reporter assay was performed, normalizing against $\beta$-galactosidase activity $(n=3)$. The luciferase activity of the GW4064 treated group was set at 100. Data are mean \pm SD. Statistical analyses were conducted using one-way ANOVA (Tukey-Kramer post hoc test). ${ }^{*} p<0.05$; ${ }^{* *} p<0.01$.

cles. After incubation for another $24 \mathrm{~h}$, the luciferase and $\beta$-galactosidase activities were measured. Normalized luciferase activities were determined by dividing the luciferase activity by the $\beta$-galactosidase activity.

Real-time quantitative PCR. Total RNA was extracted from HepG2 or the liver of mice using ISOGEN (NIPPON GENE, Tokyo, Japan), following the manufacturer's instructions. The high-capacity cDNA reverse transcrip- tion kit (Applied Biosystems, Foster, CA, USA) was used to synthesize and amplify cDNA from total RNA. Realtime quantitative PCR analyses were performed using an Applied Biosystems StepOnePlus instrument. mRNA expression was normalized to an $18 \mathrm{~S}$ ribosomal RNA (18S). Table S1 (Supplemental Online Material) describes the primers used for the real-time quantitative PCR analysis. 

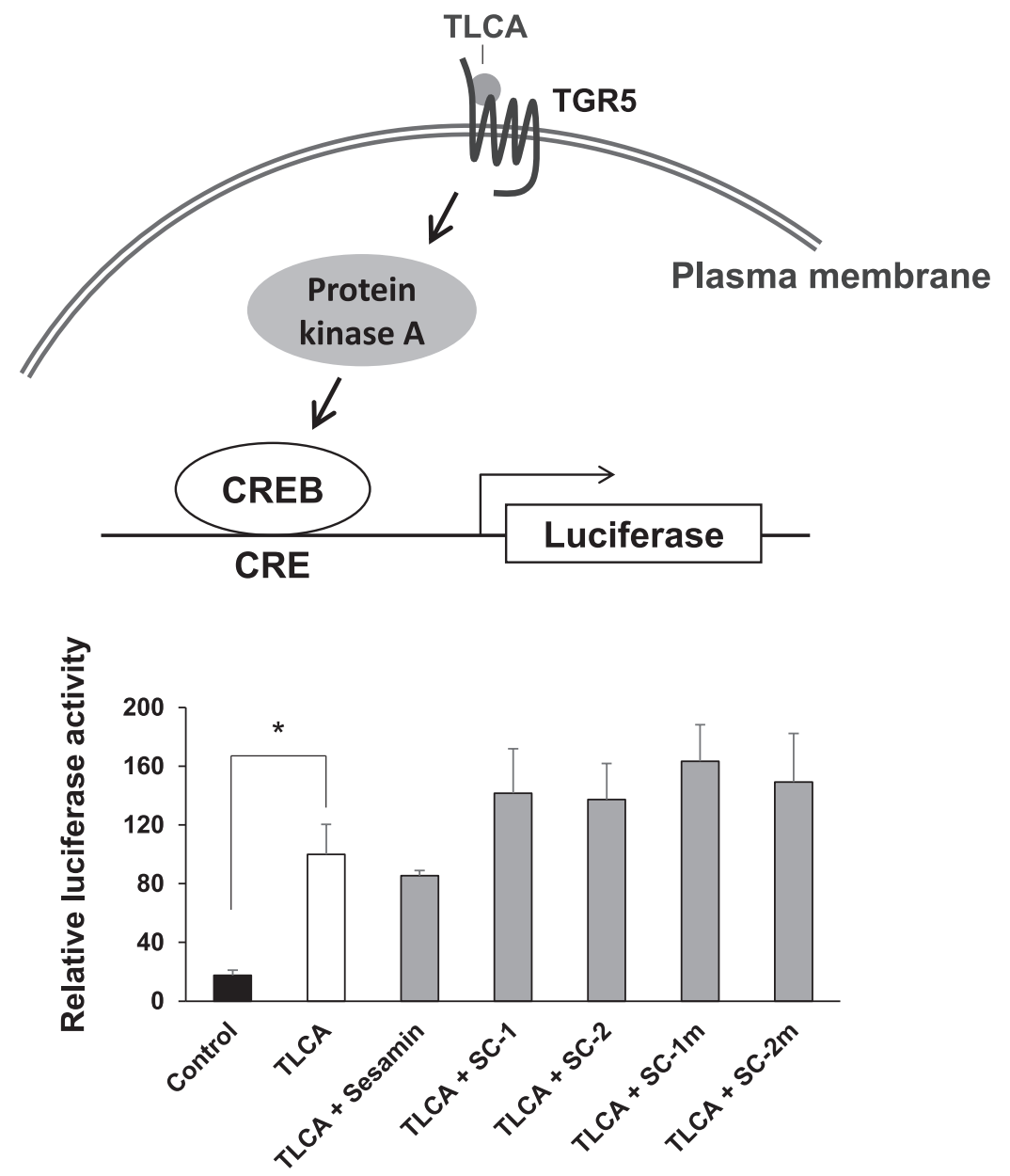

Fig. 4. Sesamin and its metabolites do not attenuate TGR5 activation by TLCA. HEK293T cells were transfected with a hTGR 5 expression plasmid together with a CRE-driven luciferase reporter plasmid. $24 \mathrm{~h}$ after transfection, the cells were cultured with TLCA $(1 \mu \mathrm{M})$ and either sesamin or its metabolites (30 $\mu \mathrm{M}$ each) for another $12 \mathrm{~h}$, and a luciferase reporter assay was performed normalizing against the $\beta$-galactosidase activity. The luciferase activity of the TLCA treated group was set at $100(n=3)$. Data are mean \pm SD. Statistical analyses were conducted using one-way ANOVA (Tukey-Kramer post hoc test). ${ }^{*} p<0.05$.

Statistical analysis. All data are presented as mean \pm SD. Two-tailed unpaired Student's $t$-tests or one-way ANOVA (Tukey-Kramer post hoc test) were used to determine $p$ values. Statistical significance was defined as $p<0.05$.

\section{RESULTS}

Sesamin, but not episesamin, reduces the I-BABP promoter activation by FXR/RXR

Sesamin has various functions, but its effects on FXR have not been clarified so far. We performed a cell-based luciferase reporter assay that evaluated FXR activity, using the HEK293T cells transfected with FXR and RXR expression plasmids, with an I-BABP promoter-luciferase plasmid to reveal the effect of sesamin and episesamin on FXR activity (Fig. 1A). We confirmed that treatment with sesamin or episesamin alone did not affect the luciferase activity in this assay system (Fig. 1B). As expected, the most potent natural FXR ligand, CDCA, increased the luciferase activity (Fig. 1B). Interestingly, sesamin suppressed CDCA-induced FXR activation significantly, but episesamin did not (Fig. 1B). These results indicate that sesamin reduces the I-BABP promoter activity regulated by FXR/RXR.

Metabolites of sesamin and episesamin antagonize FXR

In the liver, sesamin is metabolized by $\mathrm{P} 450$ and COMT, to produce SC-1, SC-2, SC-1m, and SC-2m (Fig. 2A) $(19,21)$. To evaluate the effect of these compounds on FXR activity more directly, we performed a cell-based luciferase reporter assay again using HEK293T cells transfected with an expression plasmid encoding the FXR ligand-binding domain and the GAL4 DNA-binding domain (GAL4-FXR $\mathrm{LBD}_{\text {) }}$ ) together with a UAS-luciferase plasmid. Luciferase activity was significantly increased by GW4064, a widely used synthetic agonist for the FXR, and attenuated by sesamin in a dosedependent manner; supporting our hypothesis that sesamin works as an FXR antagonist (Fig. 2B). Notably, all sesamin metabolites used in the experiments also curbed the increase in luciferase activity caused by GW4064 (Fig. 2B). These data show that not only sesamin but also its metabolites act as FXR antagonists.

Similar to sesamin, episesamin is converted into EC-1-1, EC-1-2, EC-2, EC-1m-1, EC-1m-2, EC-2m-1, 

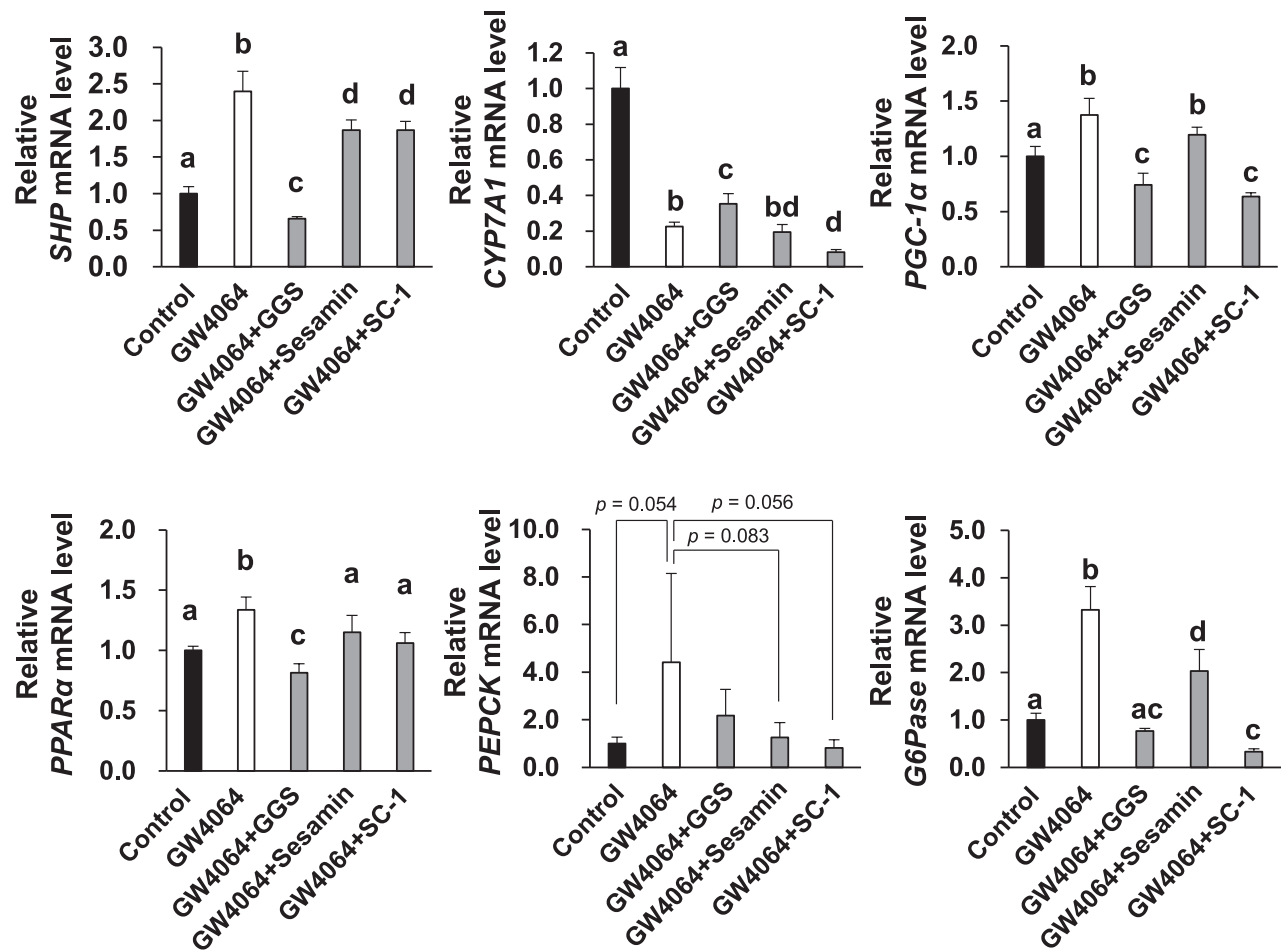

Fig. 5. Sesamin and SC-1 showed FXR antagonism in HepG2 cells. HepG2 cells were incubated in the medium containing GW4064, GW4064+GGS, GW4064+sesamin, or GW4064+SC-1 for $8 \mathrm{~h}$. The final concentration of GW4064 is $1 \mu \mathrm{M}$, and the others were $100 \mu \mathrm{M}$. The mRNA levels were determined by RT-qPCR. The data are shown as the mean \pm SD $(n=4-5)$. Different letters above the bars indicate a significant difference $(p<0.05)$ according to one-way ANOVA (TukeyKramer post hoc test).

and EC-2m-2 by P450 and COMT (Fig. 3A) (20, 21). Next, we evaluated the FXR antagonist activity of the available episesamin metabolites, EC-1-1, EC-1-2, and EC-2, in addition to episesamin. Episesamin did not affect the FXR activation driven by GW4064, whereas EC1-1 and EC1-2 suppressed it dose-dependently (Fig. 3B). EC-2 also decreased the luciferase activity significantly at $30 \mu \mathrm{M}$. However, the antagonistic effect was minor compared to other metabolites, suggesting very little FXR antagonist activity (Fig. 3B).

Sesamin and its metabolites do not antagonize the G protein-coupled bile acid receptor TGR 5

TGR 5, a G protein-coupled receptor activated by bile acids, is expressed in various tissues, and its activation exerts anti-obesity and anti-diabetic effects (22-26). Furthermore, we have reported that activation of TGR 5 in skeletal muscle induces muscle hypertrophy and improves glucose clearance in mice $(27,28)$. Therefore, it is important to investigate whether the sesamin and its metabolites antagonize TGR5 in addition to FXR. To evaluate these compounds' TGR 5 antagonistic activity, we performed a luciferase reporter assay using HEK293T cells transfected with a TGR 5 expression plasmid and a CRE-luciferase plasmid. TLCA, one of the bile acids known as the most potent TGR5 endogenous ligands, increased the luciferase activity more than 5 -fold at $1 \mu \mathrm{M}$. It was not inhibited by $30 \mu \mathrm{M}$ of sesamin and its metabolites (Fig. 4). These data indicate that sesamin and its metabolites do not affect the activation of TGR 5 .
Sesamin and SC-1 inhibit the FXR activation by GW4064 in HepG2 cells

The liver is one of the major organs that express FXR. Also, sesamin and its metabolites accumulate in the liver when sesamin is orally administered to rats (29), suggesting that the FXR antagonist activity of sesamin functions most potently in the liver. Therefore, we evaluated the antagonistic activity of sesamin and SC-1, a predominant sesamin metabolite in the liver (29), against endogenous FXR using the human hepatoma cell line HepG2 (Fig. 5). HepG2 cells treated with GW4064 for $8 \mathrm{~h}$ increased the expression of SHP, an inductive target of FXR, and decreased the expression of CYP7A1, known to be repressed by SHP. The expression of another FXR target gene, PGC- $1 \alpha$, and its downstream genes, peroxisome proliferator-activated receptor alpha (PPAR $\alpha)$, PEPCK, and G6Pase expressions, were also increased by GW4064 stimulation. Treatment of one of the FXR antagonists GGS, sesamin, and SC-1 with GW4064 significantly curbed the GW4064-induced increase in SHP expression. The inhibitory effect of sesamin and SC-1 on SHP expression was milder than GGS, and only GGS significantly increased CYP7A1 expression. The GW4064-induced increase in PGC-1 $\alpha$ gene expression was significantly suppressed by GGS and SC-1, whereas sesamin exhibited a non-significant suppressive effect. Correspondingly, the expression levels of PPAR $\alpha$ and G6Pase were suppressed considerably by GGS, sesamin, and SC-1, and the expression level of PEPCK also showed a downward trend (Fig. 5). These 
A

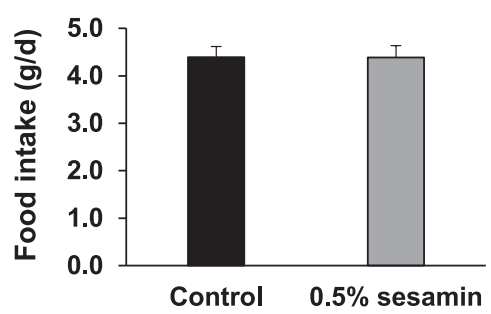

C

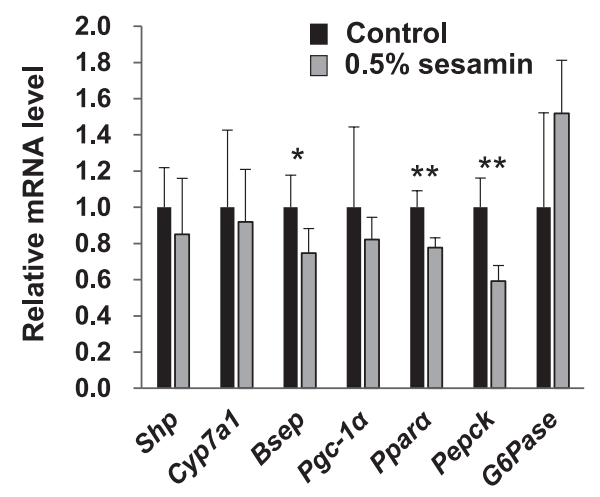

B

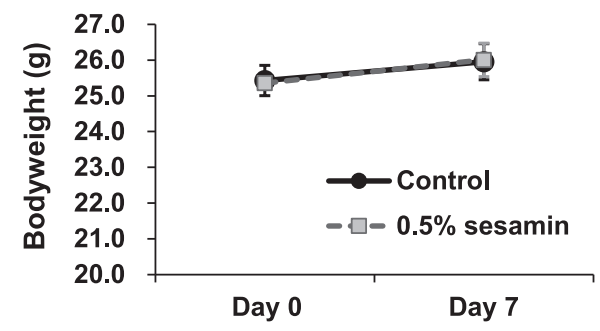

D

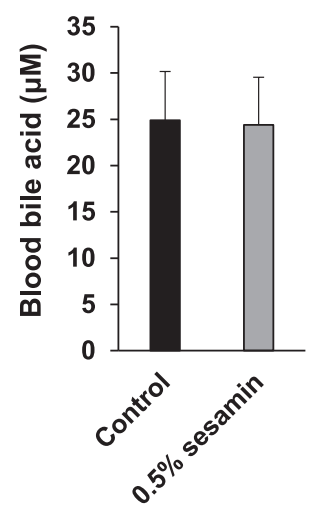

E

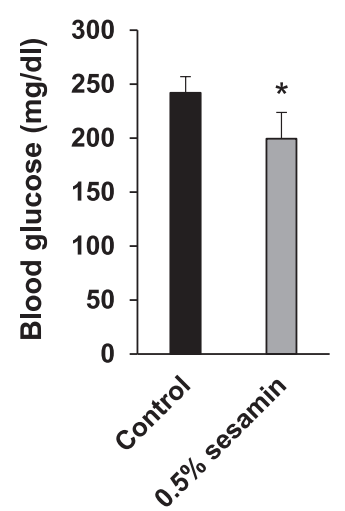

Fig. 6. Effects of $1 \mathrm{wk}$ of dietary sesamin intake on liver gene expression and blood bile acid and glucose levels in mice. Mice were fed a control diet or a $0.5 \%$ sesamin diet for $1 \mathrm{wk}(n=5)$. (A) Average food intake per day. (B) Bodyweight at the beginning and the end of the experiment. (C) The mRNA levels in the liver of each diet group. (D, E) Blood bile acid (D) and blood glucose (E) under the free-feeding condition. Data are mean \pm SD. Statistical analyses were conducted using a two-tailed unpaired Student's $t$-test. ${ }^{*} p<0.05 ;{ }^{* *} p<0.01$.

results indicate that sesamin and its predominant metabolite SC-1 can exert antagonist effects on endogenous FXR.

Sesamin supplementation suppresses gluconeogenesis gene expression and blood glucose levels in mice

Mice were fed a control diet or a $0.5 \%$ sesamin diet for 1 wk to evaluate the FXR antagonist activity of sesamin in vivo. There was no difference in food intake or bodyweight between the two groups during the 1-wk experiment (Figs. 6A and 6B). Then, liver gene expression in both groups of mice was measured. There were no significant changes in Shp and Cyp7a1 gene expression in both groups (Fig. 6C). The mRNA level of Bsep, whose expression is directly regulated by FXR (30), was significantly decreased in the $0.5 \%$ sesamin diet group (Fig. 6C). The mRNA levels of Ppar $\alpha$ and Pepck were significantly decreased by sesamin, whereas no significant changes in Pgc-1 $\alpha$ and G6Pase mRNA were observed (Fig. 6C). Next, to evaluate the effects of sesamin on bile acid and glucose metabolism, we measured blood bile acid and glucose concentration. There was no difference in the blood bile acid concentration between the two groups, whereas the blood glucose concentration was significantly decreased in the $0.5 \%$ sesamin diet group, reflecting the reduced expression of gluconeogenic genes (Figs. 6D and 6E). These results indicate that sesamin suppresses the expression of gluconeogen- esis-related genes and decreases blood glucose levels without a significant effect on bile acid metabolism.

\section{DISCUSSION}

This study first demonstrated that sesamin, sesamin metabolites, and episesamin metabolites have an FXR antagonistic activity. Although it is not clear why only episesamin is inactive despite sharing a closely resembling structure with sesamin, the fact that sesamin and its metabolites had a stronger inhibitory effect than episesamin metabolites, especially in the low concentration range, indicates that sesamin is a more potent FXR antagonist than episesamin. Because the FXR antagonist activity was evaluated by the luciferase assay using the $\mathrm{FXR}_{\mathrm{LBD}}$ in these experiments, it seems likely that these compounds exert their antagonist activity by competing with the FXR agonists on the ligand-binding domain. All of the sesamin metabolites showed FXR antagonistic activity similar to or greater than that of sesamin, with SC-1m showing the strongest. These facts indicate that sesamin has a long-term and potent FXR antagonistic activity while being metabolized by $\mathrm{P} 450$ and COMT.

Orally ingested sesamin accumulates mainly in the liver, where it is metabolized and excreted in the urine or feces (29), suggesting that the liver is the primary target organ for sesamin efficacy. Also, the liver is one of 
the major organs that express FXR. For these reasons, we tested the antagonist effect of sesamin or its metabolite SC-1 on the liver FXR using HepG2 cells. Sesamin and SC-1 significantly inhibited the increased mRNA levels of SHP, PGC-1 $\alpha$, PPAR $\alpha$, PEPCK, and G6Pase in HepG2 cells incubated with GW4064. These data indicate that sesamin and SC-1 also exert antagonist effects against endogenous FXR. Although high sesamin concentrations strongly antagonized FXR in the luciferase assay, the suppression of SHP expression by $100 \mu \mathrm{M}$ of sesamin in GW4064 treated HepG2 cells was relatively mild. This discrepancy may be partly explained by the fact that SHP gene expression is regulated by several transcription factors other than FXR. For example, p53 is known to upregulate SHP expression, which in turn regulates bile acid metabolism $(31,32)$. It is also known that treatment of hepatocytes with rifampicin, an agonist of the nuclear receptor pregnane $\mathrm{X}$ receptor (PXR), dramatically reduces the mRNA level of SHP (33). Notably, sesamin has been reported to activate p53 and also act as an antagonist of PXR (34-36), which may be why the mild suppression of SHP expression by sesamin and SC-1 in GW4064 treated HepG2 cells. Although it is unclear whether SC-1 activates p53 or antagonizes PXR so far, the comparable effect of sesamin and SC-1 on SHP gene expression implies that they may share similar functions. On the other hand, the GW4064-induced upregulation of PGC-1 $\alpha$, PEPCK, and G6Pase expression was more strongly suppressed by SC-1 than by sesamin, and the suppressive effect was equal to or greater than that of GGS. Since HepG2 cells express high levels of COMT, the SC-1 to SC-1m conversion that occurs in HepG2 cells may enhance FXR antagonism. Besides, a recent report showed that SC-1 exerts an anti-inflammatory effect through the direct binding to annexin $\mathrm{A} 1$, a property not found in sesamin, proving that SC-1 has a unique function quite different from that of sesamin (37). Collectively, it may be interesting to investigate the physiological difference between sesamin and its metabolite SC-1 in more detail.

Next, mice were fed a control diet or a $0.5 \%$ sesamin diet for $1 \mathrm{wk}$ to determine whether sesamin functions as an FXR antagonist in vivo. Dietary sesamin supplementation had no significant effect on body weight and food intake. The mRNA levels of Shp and Cyp7a1 in the liver showed a slight downward trend, and Bsep, one of the FXR target genes, was significantly downregulated. As for gluconeogenesis-related genes, Ppar $\alpha$ and Pepck were significantly downregulated. These responses of mouse liver to sesamin intake were almost consistent with those observed in sesamin-treated HepG2 cells. The reason why the expression of G6Pase mRNA was decreased by sesamin treatment in HepG2 cells, while not changed by sesamin diet in mouse liver could not be clarified in this study, but it is expected that $1 \mathrm{wk}$ of sesamin intake counteracted the reduction in G6Pase expression through some FXR-independent pathway. Despite no significant change in G6Pase gene expression upon sesamin feeding, the blood glucose levels significantly lowered with decreased expression of other glu- coneogenesis-related genes. There was no change in blood bile acid concentration between the two groups, as expected from the insignificant changes in Cyp $7 a 1$ expression. Ingested sesamin is immediately metabolized to SC-1 and SC-1m within an hour (29), and SC-1 is more potent than sesamin in suppressing the expression of gluconeogenesis-related genes induced by GW4064 in HepG2 cells. Therefore, reduced gluconeogenesis-related gene expression in liver and blood glucose levels might be due more to sesamin metabolites, including SC-1, than to sesamin itself.

Similar to FXR, the G protein-coupled receptor TGR 5 is activated by bile acids $(38,39)$. TGR 5 activation exerts anti-obesity (22-24) and anti-diabetic $(25,26)$ effects and improves skeletal muscle function $(27,28)$. Interestingly, hyocholic acid (HCA) enhances glucagon-like peptide-1 (GLP-1) secretion by inhibiting FXR activity and activating TGR5 simultaneously in the intestinal tract, thereby improving glucose metabolism in a mouse model of diabetes (40). The report also shows significantly decreased serum HCA levels in human diabetic patients, and GLP-1 levels are also decreased in correlation with HCA levels, suggesting that inactivation of FXR and activation of TGR 5 in the intestinal tract is effective in the treatment of diabetes. In this study, we observed FXR antagonistic activity in sesamin itself. Therefore, orally ingested sesamin is expected to antagonize FXR in the intestine. In addition, we also confirmed that sesamin and its metabolites do not inhibit TGR 5 activation by bile acid. Combining sesamin, an FXR antagonist, and functional food compounds such as nomilin, obacunone, oleanolic acid, and maslinic acid which have TGR 5 agonistic activity (41-45), might be an effective diabetes treatment.

In summary, we demonstrated that sesamin and its metabolites and some of the episesamin metabolites have FXR antagonist activity for the first time. Sesamin inhibits gluconeogenesis-related gene expression and lowers the blood glucose level in mice without adverse effects on bile acid metabolism. These findings reveal a new health benefit of sesamin and episesamin, which may available to improve glucose metabolism disorders.

\section{Authorship}

Research conception and design: TS and RS; experiments: MO and TWH; statistical analysis of the data: MO and TWH; interpretation of the data: TS, YW, YT, MS, YY, and RS; writing of the manuscript: TS and RS.

\section{Disclosure of state of $\mathrm{COI}$ \\ No conflicts of interest to be declared.}

\section{Acknowledgments}

We thank Dr. Manabu Horikawa (Bioorganic Research Institute, Suntory Foundation for Life Sciences) for providing sesame lignans and metabolites. We also thank Enago (Tokyo, Japan) for the English language review. 
Supporting information

Supplemental online material is available on J-STAGE.

\section{REFERENCES}

1) Parks DJ, Blanchard SG, Bledsoe RK, Chandra G, Consler TG, Kliewer SA, Stimmel JB, Willson TM, Zavacki AM, Moore DD, Lehmann JM. 1999. Bile acids: natural ligands for an orphan nuclear receptor. Science 284: 1365-1368.

2) Chiang JY, Kimmel R, Weinberger C, Stroup D. 2000. Farnesoid $\mathrm{X}$ receptor responds to bile acids and represses cholesterol 7alpha-hydroxylase gene (CYP7A1) transcription. J Biol Chem 275: 10918-10924.

3) Goodwin B, Jones SA, Price RR, Watson MA, McKee DD, Moore LB, Galardi C, Wilson JG, Lewis MC, Roth ME, Maloney PR, Willson TM, Kliewer SA. 2000. A regulatory cascade of the nuclear receptors FXR, SHP-1, and LRH-1 represses bile acid biosynthesis. Mol Cell 6: 517526.

4) Lu TT, Makishima M, Repa JJ, Schoonjans K, Kerr TA, Auwerx J, Mangelsdorf DJ. 2000. Molecular basis for feedback regulation of bile acid synthesis by nuclear receptors. Mol Cell 6: 507-515.

5) Li T, Chiang JY. 2014. Bile acid signaling in metabolic disease and drug therapy. Pharmacol Rev 66: 948-983.

6) Yamagata K, Daitoku H, Shimamoto Y, Matsuzaki H, Hirota K, Ishida J, Fukamizu A. 2004. Bile acids regulate gluconeogenic gene expression via small heterodimer partner-mediated repression of hepatocyte nuclear factor 4 and Foxo1. J Biol Chem 279: 23158-23165.

7) Magnusson I, Rothman DL, Katz LD, Shulman RG, Shulman GI. 1992. Increased rate of gluconeogenesis in type II diabetes mellitus. A $13 \mathrm{C}$ nuclear magnetic resonance study. J Clin Invest 90: 1323-1327.

8) Basu R, Chandramouli V, Dicke B, Landau B, Rizza R. 2005. Obesity and type 2 diabetes impair insulin-induced suppression of glycogenolysis as well as gluconeogenesis. Diabetes 54: 1942-1948.

9) Zhang Y, Lee FY, Barrera G, Lee H, Vales C, Gonzalez FJ, Willson TM, Edwards PA. 2006. Activation of the nuclear receptor FXR improves hyperglycemia and hyperlipidemia in diabetic mice. Proc Natl Acad Sci USA 103: 1006-1011.

10) Stayrook KR, Bramlett KS, Savkur RS, Ficorilli J, Cook T, Christe ME, Michael LF, Burris TP. 2005. Regulation of carbohydrate metabolism by the farnesoid X receptor. Endocrinology 146: 984-991.

11) Xu X, Shi X, Chen Y, Zhou T, Wang J, Chen L, Hu L, Shen X. 2018. HS218 as an FXR antagonist suppresses gluconeogenesis by inhibiting FXR binding to PGC- $1 \alpha$ promoter. Metabolism 85: 126-138.

12) Xu X, Xu X, Liu P, Zhu ZY, Chen J, Fu HA, Chen LL, Hu LH, Shen X. 2015. Structural basis for small molecule NDB (N-benzyl-N-(3-(tert-butyl)-4-hydroxyphenyl)-2,6dichloro-4-(dimethylamino) benzamide) as a selective antagonist of farnesoid X receptor $\alpha(\mathrm{FXR} \alpha)$ in stabilizing the homodimerization of the receptor. J Biol Chem 290: 19888-19899.

13) Pathak N, Rai AK, Kumari R, Bhat KV. 2014. Value addition in sesame: A perspective on bioactive components for enhancing utility and profitability. Pharmacogn Rev 8: 147-155.

14) Majdalawieh AF, Massri M, Nasrallah GK. 2017. A comprehensive review on the anti-cancer properties and mechanisms of action of sesamin, a lignan in sesame seeds (Sesamum indicum). Eur J Pharmacol 815: $512-$ 521.

15) Majdalawieh AF, Dalibalta S, Yousef SM. 2020. Effects of sesamin on fatty acid and cholesterol metabolism, macrophage cholesterol homeostasis and serum lipid profile: A comprehensive review. Eur J Pharmacol 885: 173417.

16) Li CY, Chow TJ, Wu TS. 2005. The epimerization of sesamin and asarinin. J Nat Prod 68: 1622-1624.

17) Kushiro M, Masaoka T, Hageshita S, Takahashi Y, Ide T, Sugano M. 2002. Comparative effect of sesamin and episesamin on the activity and gene expression of enzymes in fatty acid oxidation and synthesis in rat liver. J Nutr Biochem 13: 289-295.

18) Nakai M, Harada M, Nakahara K, Akimoto K, Shibata H, Miki W, Kiso Y. 2003. Novel antioxidative metabolites in rat liver with ingested sesamin. J Agric Food Chem 51: 1666-1670.

19) Yasuda K, Ikushiro S, Kamakura M, Munetsuna E, Ohta M, Sakaki T. 2011. Sequential metabolism of sesamin by cytochrome P450 and UDP-glucuronosyltransferase in human liver. Drug Metab Dispos 39: 1538-1545.

20) Tomimori N, Nakai M, Ono Y, Kitagawa Y, Kiso Y, Shibata H. 2012. Identification of the metabolites of episesamin in rat bile and human liver microsomes. Biol Pharm Bull 35: 709-716.

21) Yasuda K, Ikushiro S, Wakayama S, Itoh T, Yamamoto K, Kamakura M, Munetsuna E, Ohta M, Sakaki T. 2012. Comparison of metabolism of sesamin and episesamin by drug-metabolizing enzymes in human liver. Drug Metab Dispos 40: 1917-1926.

22) Watanabe M, Houten SM, Mataki C, Christoffolete MA, Kim BW, Sato H, Messaddeq N, Harney JW, Ezaki O, Kodama T, Schoonjans K, Bianco AC, Auwerx J. 2006. Bile acids induce energy expenditure by promoting intracellular thyroid hormone activation. Nature 439: 484-489.

23) Broeders EP, Nascimento EB, Havekes B, Brans B, Roumans KH, Tailleux A, Schaart G, Kouach M, Charton J, Deprez B, Bouvy ND, Mottaghy F, Staels B, van Marken Lichtenbelt WD, Schrauwen P. 2015. The bile acid chenodeoxycholic acid increases human brown adipose tissue activity. Cell Metab 22: 418-426.

24) Velazquez-Villegas LA, Perino A, Lemos V, Zietak M, Nomura M, Pols TWH, Schoonjans K. 2018. TGR5 signalling promotes mitochondrial fission and beige remodelling of white adipose tissue. Nat Commun 9: 245.

25) Katsuma S, Hirasawa A, Tsujimoto G. 2005. Bile acids promote glucagon-like peptide-1 secretion through TGR 5 in a murine enteroendocrine cell line STC-1. Biochem Biophys Res Commun 329: 386-390.

26) Thomas C, Gioiello A, Noriega L, Strehle A, Oury J, Rizzo G, Macchiarulo A, Yamamoto H, Mataki C, Pruzanski M, Pellicciari R, Auwerx J, Schoonjans K. 2009. TGR5-mediated bile acid sensing controls glucose homeostasis. Cell Metab 10: 167-177.

27) Sasaki T, Kuboyama A, Mita M, Murata S, Shimizu M, Inoue J, Mori K, Sato R. 2018. The exercise-inducible bile acid receptor Tgr5 improves skeletal muscle function in mice. J Biol Chem 293: 10322-10332.

28) Sasaki T, Watanabe Y, Kuboyama A, Oikawa A, Shimizu M, Yamauchi Y, Sato R. 2021. Muscle-specific TGR5 overexpression improves glucose clearance in glucose-intolerant mice. J Biol Chem 296: 100131. 
29) Tomimori N, Rogi T, Shibata H. 2017. Absorption, distribution, metabolism, and excretion of $[(14) \mathrm{C}]$ sesamin in rats. Mol Nutr Food Res $6 \mathbf{1 .}$

30) Ananthanarayanan M, Balasubramanian N, Makishima M, Mangelsdorf DJ, Suchy FJ. 2001. Human bile salt export pump promoter is transactivated by the farnesoid X receptor/bile acid receptor. J Biol Chem 276: 28857-28865.

31) Kim DH, Lee JW. 2011. Tumor suppressor $\mathrm{p} 53$ regulates bile acid homeostasis via small heterodimer partner. Proc Natl Acad Sci USA 108: 12266-12270.

32) Leblond F, Poirier S, Yu C, Duquette N, Mayer G, Thorin E. 2014. The anti-hypercholesterolemic effect of low p53 expression protects vascular endothelial function in mice. PLoS One 9: e92394.

33) Li T, Chiang JY. 2005. Mechanism of rifampicin and pregnane $\mathrm{X}$ receptor inhibition of human cholesterol 7 alpha-hydroxylase gene transcription. Am J Physiol Gastrointest Liver Physiol 288: G74-84.

34) Deng P, Wang C, Chen L, Wang C, Du Y, Yan X, Chen M, Yang G, He G. 2013. Sesamin induces cell cycle arrest and apoptosis through the inhibition of signal transducer and activator of transcription 3 signalling in human hepatocellular carcinoma cell line HepG2. Biol Pharm Bull 36: 1540-1548.

35) Kuo TN, Lin CS, Li GD, Kuo CY, Kao SH. 2020. Sesamin inhibits cervical cancer cell proliferation by promoting p53/PTEN-mediated apoptosis. Int J Med Sci 17: 22922298.

36) Lim YP, Ma CY, Liu CL, Lin YH, Hu ML, Chen JJ, Hung DZ, Hsieh WT, Huang JD. 2012. Sesamin: A naturally occurring lignan inhibits CYP3A4 by antagonizing the pregnane X receptor activation. Evid Based Complement Alternat Med 2012: 242810.

37) Kabe Y, Takemoto D, Kanai A, Hirai M, Ono Y, Akazawa S, Horikawa M, Kitagawa Y, Handa H, Rogi T, Shibata H, Suematsu M. 2020. Annexin A1 accounts for an anti-inflammatory binding target of sesamin metabolites. NPJ Sci Food 4: 4.

38) Maruyama T, Miyamoto Y, Nakamura T, Tamai Y, Okada
H, Sugiyama E, Nakamura T, Itadani H, Tanaka K. 2002. Identification of membrane-type receptor for bile acids (M-BAR). Biochem Biophys Res Commun 298: 714-719.

39) Kawamata Y, Fujii R, Hosoya M, Harada M, Yoshida H, Miwa M, Fukusumi S, Habata Y, Itoh T, Shintani Y, Hinuma S, Fujisawa Y, Fujino M. 2003. A G protein-coupled receptor responsive to bile acids. J Biol Chem 278: 9435-9440.

40) Zheng X, Chen T, Jiang R, Zhao A, Wu Q, Kuang J, Sun D, Ren Z, Li M, Zhao M, Wang S, Bao Y, Li H, Hu C, Dong B, Li D, Wu J, Xia J, Wang X, Lan K, Rajani C, Xie G, Lu A, Jia W, Jiang C, Jia W. 2021. Hyocholic acid species improve glucose homeostasis through a distinct TGR5 and FXR signaling mechanism. Cell Metab 33: 791803.e797.

41) Ono E, Inoue J, Hashidume T, Shimizu M, Sato R. 2011. Anti-obesity and anti-hyperglycemic effects of the dietary citrus limonoid nomilin in mice fed a high-fat diet. Biochem Biophys Res Commun 410: 677-681.

42) Sasaki T, Mita M, Ikari N, Kuboyama A, Hashimoto S, Kaneko T, Ishiguro M, Shimizu M, Inoue J, Sato R. 2017. Identification of key amino acid residues in the hTGR5-nomilin interaction and construction of its binding model. PLoS One 12: e0179226.

43) Horiba T, Katsukawa M, Mita M, Sato R. 2015. Dietary obacunone supplementation stimulates muscle hypertrophy, and suppresses hyperglycemia and obesity through the TGR 5 and PPARgamma pathway. Biochem Biophys Res Commun 463: 846-852.

44) Sato H, Genet C, Strehle A, Thomas C, Lobstein A, Wagner A, Mioskowski C, Auwerx J, Saladin R. 2007. Anti-hyperglycemic activity of a TGR 5 agonist isolated from Olea europaea. Biochem Biophys Res Commun 362: 793-798.

45) Murata S, Sasaki T, Yamauchi Y, Shimizu M, Sato R. 2021. Maslinic acid activates mTORC1 and human TGR 5 and induces skeletal muscle hypertrophy. Biosci Biotechnol Biochem. Advance Publication, doi:10.1093/ $\mathrm{bbb} / \mathrm{zbab} 151$. 